\title{
MINOCA Today - Are We There Yet?
}

\author{
András Mester ${ }^{1,2}$ \\ ${ }^{1}$ University of Medicine, Pharmacy, Science and Technology, Târgu Mureș, Romania \\ ${ }^{2}$ Cardiac Critical Care Unit, Clinic of Cardiology, County Clinical Emergency Hospital, Târgu Mureș, Romania
}

\section{WHAT HAVE WE ACHIEVED?}

In the last few years, the concept of myocardial infarction with non-obstructive coronary artery disease (MINOCA) has been one of the emerging hot topics in the field of cardiovascular medicine. The most recent studies in this field, focused on multiple approaches including invasive and noninvasive imaging techniques or various laboratory biomarkers, did not succeed to completely elucidate this frequent cause of acute myocardial infarction (AMI). The consensus papers published by the working groups of the European Society of Cardiology (ESC) have established the diagnostic criteria for MINOCA, defined as the absence of stenosis higher than $50 \%$ in an epicardial coronary artery at coronary angiography, in the clinical context of an AMI. At the same time, the Fourth Universal Definition of Myocardial Infarction, published in 2018, acknowledges this condition as a separate entity. ${ }^{1,2}$

\section{EMERGING EVIDENCE}

Currently, there is a lack of randomized, controlled studies on MINOCA as a separate clinical entity. However, a large number of retrospective, registry-based studies have been published recently, which highlight the importance of this entity, as it represents approximately $5-15 \%$ of all AMIs. ${ }^{3}$ Furthermore, MINOCA cannot be considered a benign condition, as the large SWEDEHEART registry-based study, which randomized 9,136 MINOCA patients with a mean follow-up period of 4 years, reported all-cause mortality rates as high as $13.5 \%$ in MINOCA patients, of which almost 50\% were declared as of cardiovascular cause. The investigators also reported important major adverse car- diovascular event (MACE) rates in MINOCA patients, with recurrent myocardial infarction in $7 \%$, ischemic stroke in $4 \%$, and hospitalization for heart failure in $6.4 \%$ of this group. ${ }^{4}$ At the same time, MINOCA patients with recurrent infarction had a $22 \%$ mortality rate at the 2-year follow-up. ${ }^{4} \mathrm{~A}$ recent large registry published by Dreyer et al., which included an impressive number of 16,849 MINOCA patients older than 65 years, reported a 25\% MACE rate at the 1-year follow-up, with lower mortality and heart failure rates compared to patients with obstructive coronary artery disease (CAD). ${ }^{5}$ Nevertheless, other recent studies suggested similar one-year survival and MACE rates as in patients with obstructive AMI. ${ }^{6-8}$ The clinical and demographic characteristics of MINOCA patients are highly heterogeneous, although systematic reviews reported younger age, high proportion of female gender (around $50 \%$ ), black race, Hispanic ethnicity, and lower prevalence of classical risk factors for CAD such as dyslipidemia, diabetes, hypertension, smoking, or family history of AMI. ${ }^{3}$

\section{IMPORTANCE OF FUNCTIONAL TESTING AND MODERN IMAGING TECHNIQUES}

As there are extensive pathophysiological mechanisms involved in the development of MINOCA, it should be considered a working diagnosis until the identification of the underlying cause. The exclusion of non-ischemic causes that might lead to similar clinical, electrocardiographic aspects or elevated cardiac enzymes (e.g., myocarditis, pulmonary embolism, sepsis) is key for the formulation of a correct final diagnosis. This involves the use of modern imaging techniques, as well as functional assessment. 
Coronary artery spasm and microvascular dysfunction are one of the most common causes of MINOCA and should be ruled out using a provocative test with acetyl choline (some studies reported a prevalence of $46 \%$ of coronary artery spasm in MINOCA patients) and determination of coronary flow reserve. ${ }^{9}$

Despite the fact that MINOCA patients do not have obstructive coronary stenoses, they can present angiographically insignificant atherosclerotic lesions. The functional assessment of these moderate $(30-50 \%)$ stenoses might be necessary, as fractional flow reserve (FFR)-based studies demonstrated that these lesions can exhibit functional significance. ${ }^{10}$ Plaque disruption is also a frequent cause of MINOCA, which can lead to thrombus formation or embolization that might not be evidenced by coronary angiography due to rapid thrombolysis. Intravascular imaging techniques such as optical coherence tomography (OCT) and intravascular ultrasound (IVUS) can identify plaque features (erosion, rupture) that may favor disruption and lead to an acute event. Studies using IVUS for the evaluation of MINOCA patients identified plaque disruption in $1 / 3$ of the examined patients. The exact prevalence of this finding is not well-established, as there is a lack of OCT-based studies, a method that is more accurate in the determination of plaque erosion and rupture due to its high resolution. OCT is also the first-line choice for the evaluation of a suspected spontaneous coronary artery dissection (SCAD), which represents an important cause of MINOCA, particularly in young women. OCT is able to identify the separation of the sub-intimal or medial layer and the presence of intramural hematoma causing significant flow obstruction, which in some cases remains unidentified (appearing as coronary wall staining, slow-flow phenomenon, or diffuse insignificant narrowing of the artery) at routine coronary angiography. ${ }^{11}$ Due to the availability and high cost of intravascular imaging techniques, the exact prevalence of SCAD in MINOCA is still yet to be elucidated. Emerging data suggests that noninvasive imaging techniques, such as coronary computed tomography angiography (CCTA), can identify SCAD in the acute setting, but this method still needs further validation. ${ }^{12}$

In the absence of coronary lesions, both angiographically as well as at intravascular imaging, cardiac magnetic resonance imaging (CMR) should be considered as the investigation of choice, being able to differentiate acute and chronic ischemic from non-ischemic myocardial lesions such as myocarditis, or Takotsubo cardiomyopathy (which does not meet the criteria of the Fourth Universal Definition of Myocardial Infarction, being discussed as a separate entity). ${ }^{1,2,13} \mathrm{~A}$ recent study published by Bhatia et al. reported positive diagnosis based on CMR in $83 \%$ of suspected MINOCA cases, while myocarditis was identified as the most common cause (32\%), followed by infarction $(22 \%)$ and non-ischemic cardiomyopathy. ${ }^{14}$

\section{CURRENT MANAGEMENT OF MINOCA}

The correct management of MINOCA is based on the identification of the underlying cause. If coronary artery spasm is identified by provocative tests, the use of calcium channel blockers and nitrates were efficient in alleviation of the symptoms and prevention of further episodes. In case of a hypercoagulable state, it is important to identify the inherited or acquired cause, and multidisciplinary approach and assessment is necessary for the initiation of chronic antiplatelet or anticoagulant therapy. ${ }^{15}$ The lack of randomized, controlled trials does create a difficult task for physicians facing this condition. The results published by the SWEDEHEART investigators support the routine use of statins, ACE inhibitors/ARBs and beta blockers, as the registry evidenced significantly lower MACE rates with these cardioprotective drugs. ${ }^{4}$ The ongoing MINOCA-BAT (Randomized Evaluation of Beta-Blocker and ACEI/ARB Treatment in MINOCA Patients) trial is one of the first randomized, placebo-controlled trials which aims to provide evidence-based medical treatment options for MINOCA patients.

\section{CONCLUSIONS}

MINOCA should be considered a working diagnosis in patients with unclear etiology of myocardial infarction, and the underlying cause of this condition should be identified for a proper management of this patient category. Modern imaging techniques can provide useful information for the understanding of the pathophysiological mechanisms that take place in the setting of a MINOCA, and randomized controlled trials are still needed to elucidate the most appropriate evidence-based treatment of this particular form of myocardial infarction.

\section{CONFLICT OF INTEREST}

Nothing to declare.

\section{REFERENCES}

1. Agewall S, Beltrame JF, Reynolds HR, et al. ESC working group position paper on myocardial infarction with non-obstructive coronary arteries. Eur Heart J. 2017;38:143-153. doi: 10.1093/ eurheartj/ehw149. 
2. Thygesen K, Alpert JS, Jaffe AS, et al. Fourth universal definition of myocardial infarction (2018). Eur Heart J. 2019;40:237-269. doi: 10.1093/eurheartj/ehy462.

3. Tamis-Holland JE, Jneid H, Reynolds HR, et al. Contemporary Diagnosis and Management of Patients With Myocardial Infarction in the Absence of Obstructive Coronary Artery Disease: A Scientific Statement From the American Heart Association. Circulation. 2019;139:e891-e908. doi: 10.1161/ CIR.0000000000000670.

4. Lindahl B, Baron T, Erlinge D, et al. Medical Therapy for Secondary Prevention and Long-Term Outcome in Patients With Myocardial Infarction With Nonobstructive Coronary Artery Disease. Circulation. 2017;135:1481-1489. doi: 10.1161/ CIRCULATIONAHA.116.026336.

5. Dreyer RP, Tavella R, Curtis JP, et al. Myocardial infarction with non-obstructive coronary arteries as compared with myocardial infarction and obstructive coronary disease: outcomes in a Medicare population. Eur Heart J. 2019;pii:ehz393. doi: 10.1093/ eurheartj/ehz403. [Epub ahead of print]

6. Safdar B, Spatz ES, Dreyer RP, et al. Presentation, clinical profile, and prognosis of young patients with myocardial infarction with nonobstructive coronary arteries (MINOCA): results from the VIRGO study. J Am Heart Assoc. 2018;pii:e009174. doi: 10.1161/JAHA.118.009174.

7. Kang WY, Jeong MH, Ahn YK, et al. Korea Acute Myocardial Infarction Registry Investigators. Are patients with angiographically near-normal coronary arteries who present as acute myocardial infarction actually safe? Int J Cardiol. 2011;146:207-212. doi: 10.1016/j.ijcard.2009.07.001.

8. Abdu FA, Liu L, Mohammed AQ, et al. Myocardial infarction with non-obstructive coronary arteries (MINOCA) in Chinese patients: Clinical features, treatment and 1 year follow-up. Int J Cardiol. 2019;287:27-31. doi: 10.1016/j.ijcard.2019.02.036.
9. Montone RA, Niccoli G, Fracassi F, et al. Patients with acute myocardial infarction and nonobstructive coronary arteries: safety and prognostic relevance of invasive coronary provocative tests. Eur Heart J. 2018;39:91-98. doi: 10.1093/ eurheartj/ehx667.

10. Garcia D, Harbaoui B, van de Hoef TP, et al. Relationship between FFR, CFR and coronary microvascular resistance Practical implications for FFR-guided percutaneous coronary intervention. PLoS One. 2019;14:e0208612. doi:10.1371/journal. pone.0208612.

11. Pasupathy S, Air T, Dreyer RP, Tavella R, BeltrameJF. Systematic review of patients presenting with suspected myocardial infarction and nonobstructive coronary arteries. Circulation. 2015;131:861-70. doi: 10.1161/CIRCULATIONAHA.114.011201.

12. Tweet MS, Akhtar NJ, Hayes SN, Best PJ, Gulati R, Araoz PA. Spontaneous coronary artery dissection: Acute findings on coronary computed tomography angiography. Eur Heart J Acute Cardiovasc Care. 2019;8:467-475. doi: 10.1177/2048872617753799.

13. Pathik B, Raman B, Mohd Amin NH, et al. Troponin-positive chest pain with unobstructed coronary arteries: incremental diagnostic value of cardiovascular magnetic resonance imaging. Eur Heart J Cardiovasc Imaging. 2016;17:1146-1152. doi: 10.1093/ehjci/jev289.

14. Bhatia S, Anstine C, Jaffe AS, et al. Cardiac magnetic resonance in patients with elevated troponin and normal coronary angiography. Heart 2019;105:1231-1236. doi: 10.1136/ heartjnl-2018-314631.

15. Mukherjee D. Myocardial Infarction With Nonobstructive Coronary Arteries: A Call for Individualized Treatment. J Am Heart Assoc. 2019;8:e013361. doi: 10.1161/JAHA.119.013361. 